\title{
Defect production due to quenching through a multicritical point and along a gapless line
}

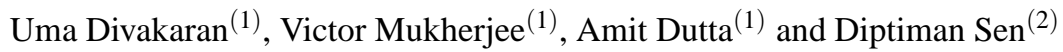 \\ (1) Department of Physics, Indian Institute of Technology Kanpur, Kanpur 208 016, India \\ (2) Center for High Energy Physics, Indian Institute of Science, Bangalore 560 012, India
}

\subsection{Introduction}

The exciting physics of quantum phase transitions has been explored extensively in the last few years [1, 2]. The non-equilibrium dynamics of a quantum system when quenched very fast [3] or slowly across a quantum critical point [4, 5] has attracted the attention of several groups recently. The possibility of experimental realizations of quantum dynamics in spin-1 Bose condensates [6] and atoms trapped in optical lattices [7, 8] has led to an upsurge in studies of related theoretical models [3, 4, 5, 9 , 10, 11, 12, 13, 14, 15, 16, 17, 18, 19, 20, 21, 23, 24, 25, 26, 27, 28, 29, 30, 31, 32, 33].

In this review, we concentrate on the dynamics of quantum spin chains swept across a quantum critical or multicritical point or along a gapless line by a slow (adiabatic) variation of a parameter appearing in the Hamiltonian of the system. Our aim is to find the scaling form of the density of defects (which, in our case, is the density of wrongly oriented spins) in the final state which is reached after the system is prepared in an initial ground state and then slowly quenched through a quantum critical point. The dynamics in the vicinity of a quantum critical point is necessarily non-adiabatic due to the divergence of the relaxation time of the underlying quantum system which forces the system to be infinitely sluggish; thus the system fails to respond to a change in a parameter of the Hamiltonian no matter how slow that rate of change may be!

We first recall the Kibble-Zurek argument [34, 35] which predicts a scaling form for the defect density following a slow quench through a quantum critical point. We assume that a parameter $g$ of the Hamiltonian is varied in a linear fashion such that $g-g_{c} \sim t / \tau$, where $g=g_{c}$ denotes the value of $g$ at the quantum critical point and $\tau$ is the quenching time. Our interest is in the adiabatic limit, $\tau \rightarrow \infty$. The energy gap of the quantum Hamiltonian vanishes at the critical point as $\left(g-g_{c}\right)^{v z}$ whereas the relaxation time $\xi_{\tau}$, which is inverse of the gap, diverges at the critical point. It is clear that non-adiabaticity becomes important at a time $\hat{t}$ when the characteristic time scale of the quantum system (i.e., the relaxation time) is of the order of the inverse of the rate of change of the Hamiltonian; this yields 
This leads to a characteristic length scale $\xi$ given by

$$
\xi \sim \tau^{-v /(v z+1)}
$$

where $\xi$ is the correlation length or healing length. The healing length typically denotes the length over which a single defect is present. The density of defects in a $d$-dimensional system scales as $1 / \xi^{d}$ which leads to the Kibble-Zurek scaling form for the density of defects $n$ given by

$$
n \sim \tau^{-d v /(v z+1)} .
$$

The interesting aspect of the Kibble-Zurek prediction is that the scaling form of the defect density in the final state of a driven quantum system varies in a power-law fashion with the rate of quenching $1 / \tau$, and the exponent of the power-law depends on the spatial dimension $d$ and the static quantum critical exponents $v$ and $z$.

The Kibble-Zurek scaling has been verified in various exactly solvable spin models and systems of interacting bosons [4, 13, 16, 17, 11, 5]; it has been generalized to quenching through a multicritical point [31], across a gapless phase [20, 23], and along a gapless line [26, 28], and to systems with quenched disorder [14], white noise [18], infinite-range interactions [27], and edge states [30]. Studies have also been made to estimate the defect density for quenching with a non-linear form [21], an oscillatory variation of an applied magnetic field [32] or under a reversal of the magnetic field [33]. In the article in this book by Mondal, Sengupta and Sen [36], the quenching dynamics through a gapless phase and quenching with a power-law form of the change of a parameter as well as the possibility of experimental realizations have been discussed in detail. It should be mentioned that in addition to the density of defects in the final state, the degree of non-adiabaticity can also be quantified by looking at various quantities like residual energy [9, 37] and fidelity [4].

\subsection{A spin model: transverse and anisotropic quenching}

In this article, we will focus mainly on the one-dimensional anisotropic $X Y$ spin-1/2 chain in a transverse field [38, 39] which is represented by the Hamiltonian

$$
H=-\sum_{n}\left[J_{x} \sigma_{n}^{x} \sigma_{n+1}^{x}+J_{y} \sigma_{n}^{y} \sigma_{n+1}^{y}+h \sigma_{n}^{z}\right]
$$

where $\sigma^{\prime} s$ are the usual Pauli matrices. (We will set Planck's constant $\hbar=1$ ). The spectrum of this Hamiltonian can be found exactly by first mapping the $\sigma$ matrices to Jordan-Wigner fermions $c_{n}$ as [39]

$$
\begin{aligned}
& c_{n}=\sigma_{n}^{-} \exp \left(i \pi \sum_{j=1}^{n-1} \sigma_{j}^{+} \sigma_{j}^{-}\right), \\
& \sigma_{n}^{z}=2 c_{n}^{\dagger} c_{n}-1,
\end{aligned}
$$


where $\sigma_{n}^{ \pm}=\sigma_{n}^{x} \pm i \sigma_{n}^{y}$ are spin raising and lowering operators respectively. These Jordan-Wigner fermion operators follow the usual anticommutation rules

$$
\left\{c_{m}^{\dagger}, c_{n}\right\}=\delta_{m n} ; \quad\left\{c_{m}, c_{n}\right\}=0=\left\{c_{m}^{\dagger}, c_{n}^{\dagger}\right\} .
$$

Applying a Fourier transformation to the Jordan-Wigner fermions along with the periodic boundary conditions, the Hamiltonian in (1.4) can be rewritten as

$$
\begin{aligned}
H=-\sum_{k>0}\{ & {\left[\left(J_{x}+J_{y}\right) \cos k+h\right]\left(c_{k}^{\dagger} c_{k}+c_{-k}^{\dagger} c_{-k}\right) } \\
& +i\left(J_{x}-J_{y}\right) \sin k\left(c_{k}^{\dagger} c_{-k}^{\dagger}-c_{-k} c_{k}\right\},
\end{aligned}
$$

where $k$ lies in the range $[0, \pi]$. The Hamiltonian is quadratic in fermion operators and hence exactly solvable using an appropriate Bogoliubov transformation [39]. Using the basis vectors $|0\rangle$ (where no fermions are present) and $|k,-k\rangle=c_{k}^{\dagger} c_{-k}^{\dagger}|0\rangle$, we can recast the Hamiltonian for wave number $k$ in a $2 \times 2$ matrix form

$$
H_{k}=\left[\begin{array}{ll}
h+\left(J_{x}+J_{y}\right) \cos k & i\left(J_{x}-J_{y}\right) \sin k \\
-i\left(J_{x}-J_{y}\right) \sin k & -h-\left(J_{x}+J_{y}\right) \cos k
\end{array}\right]
$$

The spectrum for this is given by

$$
\varepsilon_{k}=\left[h^{2}+J_{x}^{2}+J_{y}^{2}+2 h\left(J_{x}+J_{y}\right) \cos k+2 J_{x} J_{y} \cos 2 k\right]^{1 / 2} .
$$

This Hamiltonian has a very rich phase diagram as shown in Fig. 1.1. The vanishing of the energy gap for $h= \pm\left(J_{x}+J_{y}\right)$ for the critical modes at $k=\pi$ and 0 , respectively, signals quantum phase transitions from a ferromagnetically ordered phase to a paramagnetic phase with critical exponents $v=z=1$ [38]. On the other hand, the vanishing energy gap at $J_{x}=J_{y}$ and $h<J_{x}+J_{y}$ denotes the anisotropic transition which marks the boundary between the two ferromagnetic phases with the critical exponents $v$ and $z$ being identical to the Ising transition. The meeting points of these two transition lines at $h= \pm\left(J_{x}+J_{y}\right)$ and $J_{x}=J_{y}$ are multicritical points.

Let us initiate our discussions with two types of quenching schemes:

(i) quenching the magnetic field $h$ as $t / \tau$ which we call transverse quenching [11, 13], and

(ii) the quenching of the interaction $J_{x}$ as $t / \tau$ which is referred to as anisotropic quenching [16].

In the process of anisotropic quenching, the system can be made to cross the multicritical points $A$ and $B$ shown in the phase diagram. The quenching through a multicritical point is a cardinal point of our discussion. We shall also discuss the quenching scheme where the anisotropy parameter $\gamma=J_{x}-J_{y}$ is quenched in a linear fashion keeping the system always on the gapless line $h=J_{x}+J_{y}$. It will be shown that in either case, we arrive at new scaling behaviors for the defect density which cannot be obtained by a simple fine tuning of the Kibble-Zurek scaling form $n \sim 1 / \tau^{v d / v z+1}$.

The reduction of the general Hamiltonian to a direct product of $2 \times 2$ matrices as given in (1.8) facilitates the application of the Landau-Zener (LZ) transition formula [40] to estimate the non-adiabatic transition probability on passing through a quantum critical point. The general LZ Hamiltonian given by 


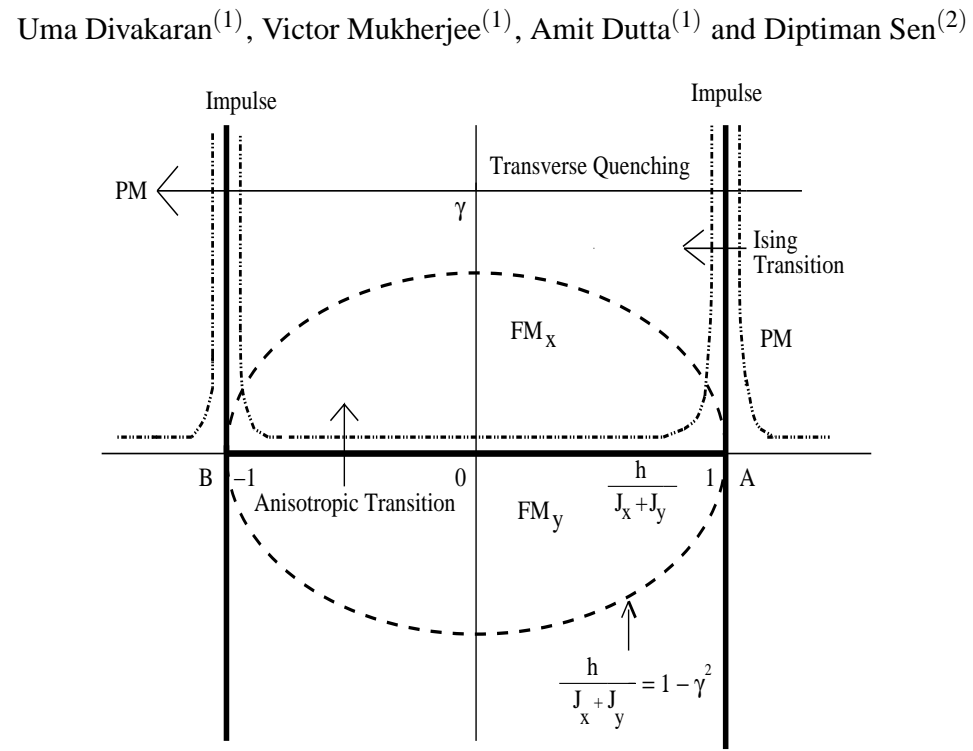

Fig. 1.1. The phase diagram of the anisotropic $X Y$ model in a transverse field in the $h /\left(J_{x}+\right.$ $\left.J_{y}\right)-\gamma$ plane, where $\gamma \equiv\left(J_{x}-J_{y}\right) /\left(J_{x}+J_{y}\right)$. The vertical bold lines given by $h /\left(J_{x}+J_{y}\right)=$ \pm 1 denote the Ising transitions. The system is also gapless on the horizontal bold line $\gamma=0$ for $|h|<J_{x}+J_{y} . \mathrm{FM}_{\mathrm{x}}\left(\mathrm{FM}_{\mathrm{y}}\right)$ is a long-range ordered phase with ferromagnetic ordering in the $x(y)$ direction. The thick dashed line marks the boundary between the commensurate and incommensurate ferromagnetic phases. The thin dotted lines indicate the adiabatic and impulse regions when the field $h$ is quenched from $-\infty$ to $\infty$. The two points with coordinates $\gamma=0$ and $h /\left(J_{x}+J_{y}\right)= \pm 1$ denoted by $A$ and $B$ are the multicritical points.

$$
H=\varepsilon_{1}|1\rangle\left\langle 1\left|+\varepsilon_{2}\right| 2\right\rangle\langle 2|+\Delta(|1\rangle\langle 2|+| 2\rangle\langle 1|)
$$

closely resembles the reduced $2 \times 2$ spin Hamiltonian given in (1.8). In the LZ Hamiltonian, we set $\varepsilon_{1}-\varepsilon_{2}=t / \tau$. The two energy levels $\pm \sqrt{\varepsilon^{2}+\Delta^{2}}$, where $\varepsilon_{1}=-\varepsilon_{2}=\varepsilon$, approach each other with a minimum gap $2 \Delta$ at $t=0$ as shown in Fig. 1.2. The system is prepared in its initial ground state $|1\rangle$ at time $t \rightarrow-\infty$ and should reach the final ground state $|2\rangle$ at $t \rightarrow+\infty$ if the dynamics is adiabatic throughout. A general state during the time evolution can be written in the form $|\psi(t)\rangle=C_{1}(t)|1\rangle+C_{2}(t)|2\rangle$ with the initial condition $\left|C_{1}(t \rightarrow-\infty)\right|^{2}=1$. The $\mathrm{LZ}$ probability for the non-adiabatic transition is given by [40, 37]

$$
p=\left|C_{1}(t \rightarrow \infty)\right|^{2}=e^{-2 \pi \Delta^{2} /\left|\frac{\partial}{\partial t}\left(\varepsilon_{1}-\varepsilon_{2}\right)\right|} .
$$

It is to be noted that the above formula is valid for a linear variation of the bare levels $\varepsilon_{1}$ and $\varepsilon_{2}$ and also when the off-diagonal term does not include any time dependence.

Let us now discuss the transverse quenching of the $X Y$ spin chain discussed above. The phase diagram in (1.1) shows that when the transverse field is quenched from $-\infty$ to $+\infty$, the system is swept across the two Ising transition lines at $h=$ $\pm\left(J_{x}+J_{y}\right)$. Referring to the Hamiltonian in (1.8), it is clear that when $h$ is quenched 


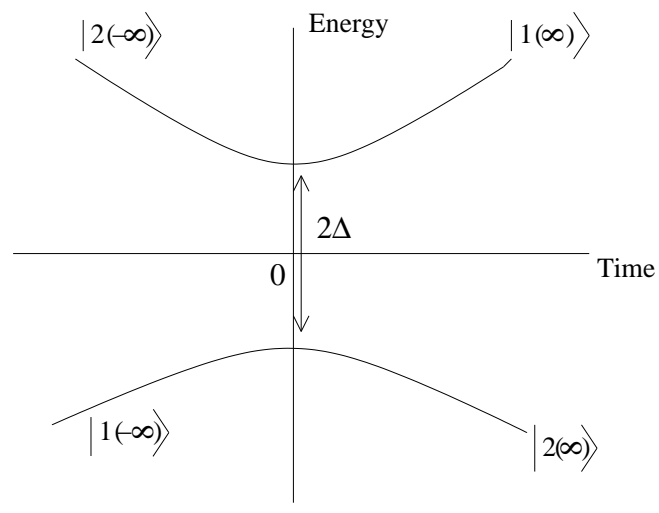

Fig. 1.2. The two levels correspond to the energy levels varying with time; $2 \Delta$ is the minimum gap between these two levels, and $|1\rangle$ and $|2\rangle$ are the eigenstates in the asymptotic limits $t \rightarrow \pm \infty$. The ground state changes its characteristics from $|1\rangle$ at $t \rightarrow-\infty$ to $|2\rangle$ at $t \rightarrow+\infty$.

as $h \sim t / \tau$ [13], the dynamics of the spin chain effectively reduces to a LZ problem in the two-dimensional reduced Hilbert space spanned by the basis vectors $|0\rangle$ and $|k,-k\rangle$, with the initial ground state being $|0\rangle$. Here, the diagonal and off-diagonal terms are denoted by $\varepsilon_{k}^{ \pm}= \pm\left[h+\left(J_{x}+J_{y}\right) \cos k\right]$ and $\Delta=i\left(J_{x}-J_{y}\right) \sin k$ respectively. Denoting a general state vector $\psi_{k}(t)$ at an instant $t$ as $\left|\psi_{k}(t)\right\rangle=C_{1, k}|0\rangle+C_{2, k}|k,-k\rangle$ with $\left|C_{1, k}(t \rightarrow-\infty)\right|^{2}=1$, the non-adiabatic transition probability for the mode $k$ is directly obtained using the LZ transition formula

$$
p_{k}=\left|C_{1, k}(t \rightarrow \infty)\right|^{2}=e^{-\pi \tau\left|J_{x}-J_{y}\right|^{2} \sin ^{2} k} .
$$

The density of defects (i.e., density of wrongly oriented spins) in the final state is obtained by integrating $p_{k}$ over the Brillouin zone [13],

$$
n=\frac{1}{2 \pi} \int_{-\pi}^{\pi} p_{k} d k \approx \frac{1}{\pi \sqrt{\tau}\left(J_{x}-J_{y}\right)} .
$$

In the adiabatic limit $\tau \rightarrow \infty$, the transition probability is non-zero only for modes close to the critical modes $k=0$ and $\pi$; this allows us to extend the limits of integration from $-\infty$ to $+\infty$. Noting that the critical exponents $v=z=1$ for the Ising transition and $d=1$; hence the $1 / \sqrt{\tau}$ scaling of the defect density is consistent with the Kibble-Zurek prediction.

On the other hand, if we look at the anisotropic quenching $J_{x} \sim t / \tau$, the offdiagonal terms of the Hamiltonian in (1.8) pick up a time dependence and hence a direct application of the LZ transition probability is not possible. To overcome this problem, we rewrite the Hamiltonian in the basis of the initial and final eigenstates when $J_{x} \rightarrow-\infty$ and $J_{x} \rightarrow \infty$. The eigenstates of the Hamiltonian in these limits are given by

$$
\left|e_{1 k}\right\rangle=\sin (k / 2)|0\rangle+i \cos (k / 2)|k,-k\rangle
$$


and

$$
\left|e_{2 k}\right\rangle=\cos (k / 2)|0\rangle-i \sin (k / 2)|k,-k\rangle,
$$

with eigenvalues $\lambda_{1}=t / \tau$ and $\lambda_{2}=-t / \tau$ respectively; the system is in the state $\left|e_{1 k}\right\rangle$ initially. A general state vector can be expressed as a linear combination of $\left|e_{1 k}\right\rangle$ and $\left|e_{2 k}\right\rangle$,

$$
\left|\psi_{k}(t)\right\rangle=C_{1 k}(t)\left|e_{1 k}\right\rangle+C_{2 k}(t)\left|e_{2 k}\right\rangle .
$$

The initial condition in the anisotropic case is $C_{1 k}(-\infty)=1$ and $C_{2 k}(-\infty)=0$. The unitary transformation to rewrite the Hamiltonian in the $\left|e_{1 k}\right\rangle$ and $\left|e_{2 k}\right\rangle$ basis is given by $H_{k}^{\prime}(t)=U^{\dagger} H_{k}(t) U$, where

$$
U=\left[\begin{array}{cc}
\cos (k / 2) & \sin (k / 2) \\
-i \sin (k / 2) & i \cos (k / 2)
\end{array}\right]
$$

and the new Hamiltonian is

$$
\begin{aligned}
H_{k}^{\prime}(t)= & -\left[h+\left(J_{x}+J_{y}\right) \cos k\right] I_{2} \\
& +\left[\begin{array}{cc}
J_{x}+J_{y} \cos 2 k+h \cos k & J_{y} \sin 2 k+h \sin k \\
J_{y} \sin 2 k+h \sin k & -J_{x}-J_{y} \cos 2 k-h \cos k
\end{array}\right] .
\end{aligned}
$$

By virtue of the unitary transformation, the time dependence is entirely shifted to the diagonal terms which makes it possible to apply the LZ transition formula. Evaluating the probability of a non-adiabatic transition for the mode $k$ and integrating over the Brillouin zone, it can be shown that the density of defects in the final state following the anisotropic quenching is given by [16]

$$
\begin{aligned}
n & \sim \frac{4 J_{y}}{\pi \sqrt{\tau}\left(4 J_{y}^{2}-h^{2}\right)} \quad \text { for } h<2 J_{y}, \\
& \sim \frac{h}{\pi \sqrt{\tau}\left(h^{2}-4 J_{y}^{2}\right)} \quad \text { for } h>2 J_{y} .
\end{aligned}
$$

The scaling behavior of the density of defects with $\tau$ is shown in Fig. 1.3 .

Although Eqs. (1.16) satisfy the Kibble-Zurek scaling, the density of defects diverges for $h=2 J_{y}$, i.e., in a passage through the multicritical points. This necessitates a generalization of the Kibble-Zurek prediction for quenching through a multicritical point. In the next section, we propose a generalized scaling for the density of defects valid for quenching through a critical point as well as a multicritical point.

In Fig. 1.4, we present the variation of the von Neumann entropy density of the final state defined by [13]

$$
s=-\int_{0}^{\pi} \frac{d k}{\pi}\left[p_{k} \ln \left(p_{k}\right)+\left(1-p_{k}\right) \ln \left(1-p_{k}\right)\right] .
$$

following the anisotropic quenching with the rate of quenching. Even though the final state is a pure state as a result of a unitary dynamics, it can also be viewed locally, or on a coarse-grained wave vector scale, as a decohered (mixed) state [13]. In the limit 


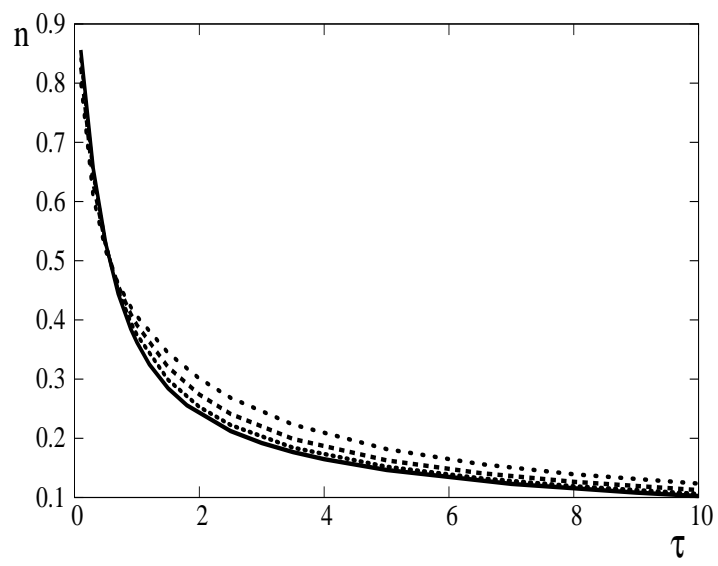

Fig. 1.3. Plot of kink density $n$ versus $\tau$ as obtained for $h=0.2,0.4,0.6,0.8$ (from bottom to top in the large $\tau$ region), with $J_{y}=1$ for anisotropic quenching. For large $\tau, n$ increases with increasing $h$, whereas for small $\tau$, it decreases with increasing $h$.

of $\tau \rightarrow 0$, the system does not get enough time to evolve; hence it largely retains its initial antiferromagnetic order which results in a low local entropy density. On the other hand, for slow quenching $(\tau \rightarrow \infty)$, the system evolves adiabatically, always remaining close to its instantaneous ground state; this results in a final state with local ferromagnetic ordering, and hence again with a low local entropy density. We observe that the entropy density shows a maximum at a characteristic time scale $\tau_{0}$ where the magnetic ordering of the final state also changes from antiferromagnetic to ferromagnetic.

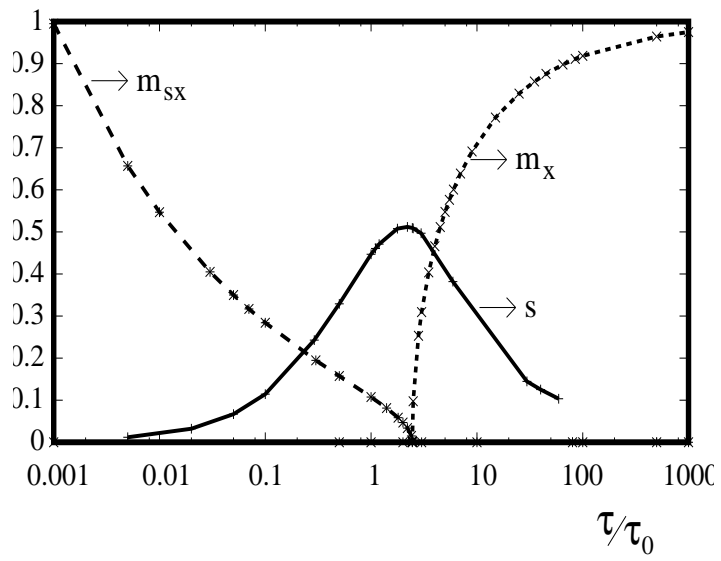

Fig. 1.4. Plot of von Neumann entropy density $s$, staggered magnetization $m_{s x}$ and magnetization $m_{x}$ as a function of $\tau / \tau_{0}$, for $J_{y}=1$ and $h=0.2$ in the anisotropic quenching case. 
Let us briefly mention a few other interesting variants of the transverse quenching scheme. In a repeated quenching dynamics of the same model, the transverse field $h(=t / \tau)$ is quenched repeatedly between $-\infty$ and $+\infty$ [25]. We refer to a single passage from $h \rightarrow-\infty$ to $h \rightarrow+\infty$ or the other way around as a half-period of quenching. An even number of half-periods corresponds to the transverse field being brought back to its initial value of $-\infty$, whereas, in the case of an odd number of half-periods, the dynamics is stopped at $h \rightarrow+\infty$. The probability of a non-adiabatic transition at the end of $l$ half-periods can be shown to follow the recursion relation

$$
\left.p_{k}(l)=\left(1-e^{-2 \pi \gamma}\right)-\left(1-2 e^{-2 \pi \gamma}\right)\left[1-p_{k}(l-1)\right)\right],
$$

eventually yielding the simplified form of

$$
p_{k}(l)=\frac{1}{2}-\frac{\left(1-2 e^{-2 \pi \gamma}\right)^{l}}{2} .
$$

For large $\tau$, the density of defects is generally found to vary as $1 / \sqrt{\tau}$ for any number of half-periods. On the other hand, for small $\tau$, it shows an increase in kink density for even values of $l$. However, the magnitude is found to depend on the number of half-periods of quenching. For two successive half-periods, the defect density is found to decrease in comparison to a single half-period, suggesting the existence of a corrective mechanism in the reverse path. The entropy density increases monotonously with the number of half-periods, and shows qualitatively the same behavior for any number of half-periods. For a large number of repetitions $(l \rightarrow \infty)$, the defect density saturates to the value $1 / 2$ while the local entropy density saturates to $\ln 2$.

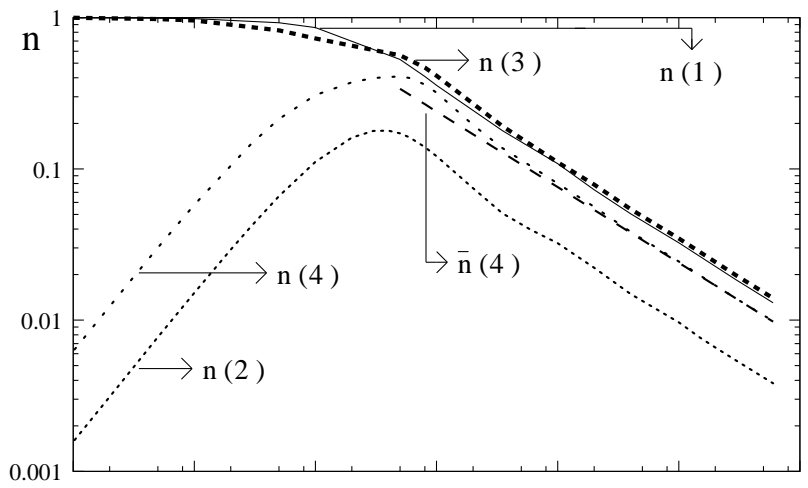

Fig. 1.5. Plot of kink density $n$ after $l$ half cycles as a function of $\tau$, for $J_{x}-J_{y}=1$ and $l=1,2,3,4 . \bar{n}(4)$ denotes the defect density as obtained from the analytical expression. In the limit of large $\tau$, the numerical results match perfectly with the analytical results.

The effects of interference on the quenching dynamics of Hamiltonian in 1.8 when the transverse field $h(t)$ varies sinusoidally with time as $h=h_{0} \cos \omega t$, with 
$|t| \leq \pi / \omega$ has also been studied in a recent work [32]. In this scheme of quenching, the time interval between two successive passages through the quantum critical points can be small enough for the presence of non-trivial effects of interference in the dynamics of the system. It has been shown that for a single passage through a quantum critical point, the interference effects do not contribute leading to a kink density which goes as $n \sim \sqrt{\omega}$. On the other hand, repeated passages through the quantum critical points result in an oscillatory behavior of the kink density as well as the entropy density.

\subsection{Quenching through a multicritical point}

We shall now propose a general scaling scheme valid for quenching through a multicritical point as well as a critical point [31] using the LZ non-adiabatic transition probability [40, 37] discussed before. We begin with a generic $d$-dimensional model Hamiltonian of the form

$$
H=\sum_{\mathbf{k}} \psi^{\dagger}(\mathbf{k})\left[(\lambda(t)+b(\mathbf{k})) \sigma^{z}+\Delta(\mathbf{k}) \sigma^{+}+\Delta^{*}(\mathbf{k}) \sigma^{-}\right] \psi(\mathbf{k}),
$$

where $\sigma^{ \pm}=\sigma^{x} \pm i \sigma^{y}, b(\mathbf{k})$ and $\Delta(\mathbf{k})$ are model dependent functions, and $\psi(\mathbf{k})$ denotes the fermionic operators $\left(\psi_{1}(\mathbf{k}), \psi_{2}(\mathbf{k})\right)$. The above Hamiltonian represents, for example, a one-dimensional transverse Ising or $X Y$ spin chain [39] as shown in Eq. (1.8), or an extended Kitaev model in $d=2$ written in terms of Jordan-Wigner fermions [41, 20]. The excitation spectrum takes the form

$$
\varepsilon_{k}^{ \pm}= \pm \sqrt{(\lambda(t)+b(\mathbf{k}))^{2}+|\Delta(\mathbf{k})|^{2}} .
$$

We assume that the parameter $\lambda(t)$ varies linearly as $t / \tau$ and vanishes at $t=0$. The parameters $b(\mathbf{k})$ and $\Delta(\mathbf{k})$ are assumed to vanish at the quantum critical point in a power-law fashion given by

$$
b(\mathbf{k}) \sim|\mathbf{k}|^{z_{1}} \text { and } \Delta(\mathbf{k}) \sim|\mathbf{k}|^{z_{2}},
$$

where we have taken the critical mode to be at $\mathbf{k}_{0}=0$ without any loss of generality. Eq. (1.22) also implies that the system crosses the gapless point at $t=0$ when $\lambda=0$, and $b(\mathbf{k})$ and $\Delta(\mathbf{k})$ also vanish for the critical mode $\mathbf{k}=0$. Many of the models described by Eq. (1.20) exhibit a quantum phase transition with the exponents associated with the quantum critical point being $z_{1}>z_{2}$ and hence $z=z_{2}=1$. We shall however explore the more general case encountered at a multicritical point where the dynamically exponent $z$ is not necessarily given by $z_{2}$.

The Schrödinger equation describing the time evolution of the system when $\lambda$ is quenched is given by $i \partial \psi_{\mathbf{k}} / \partial t=H \psi_{\mathbf{k}}$, where we have once again defined $\psi_{\mathbf{k}}$ as $\psi_{\mathbf{k}}=\tilde{C}_{1, \mathbf{k}}|0\rangle+\tilde{C}_{2, \mathbf{k}}|\mathbf{k},-\mathbf{k}\rangle$. Using the Hamiltonian in Eq. (1.20), we can write

$$
\begin{aligned}
& i \frac{\partial \tilde{C}_{1, \mathbf{k}}}{\partial t}=\left(\frac{t}{\tau}+b(\mathbf{k})\right) \tilde{C}_{1, \mathbf{k}}+\Delta(\mathbf{k}) \tilde{C}_{2, \mathbf{k}}, \\
& i \frac{\partial \tilde{C}_{2, \mathbf{k}}}{\partial t}=-\left(\frac{t}{\tau}+b(\mathbf{k})\right) \tilde{C}_{2, \mathbf{k}}+\Delta^{*}(\mathbf{k}) \tilde{C}_{1, \mathbf{k}} .
\end{aligned}
$$


One can now remove $b(\mathbf{k})$ from the above equations by redefining $t / \tau+b(\mathbf{k}) \rightarrow t / \tau$; thus the exponent $z_{1}$ defined in Eq. (1.22) does not play any role in the following calculations. Defining a new set of variables $C_{1, \mathbf{k}}=\tilde{C}_{1, \mathbf{k}} \exp \left(i \int^{t} d t^{\prime} t^{\prime} / \tau\right)$ and $C_{2, \mathbf{k}}=$ $\tilde{C}_{2, \mathbf{k}} \exp \left(-i \int^{t} d t^{\prime} t^{\prime} / \tau\right)$, we arrive at a time evolution equation for $C_{1}(\mathbf{k})$ given by

$$
\left(\frac{d^{2}}{d t^{2}}-2 i \frac{t}{\tau} \frac{d}{d t}+|\Delta(\mathbf{k})|^{2}\right) C_{1, \mathbf{k}}=0 .
$$

Further rescaling $t \rightarrow t \tau^{1 / 2}$ leads to

$$
\left(\frac{d^{2}}{d t^{2}}-2 i t \frac{d}{d t}+|\Delta(\mathbf{k})|^{2} \tau\right) C_{1, \mathbf{k}}=0 .
$$

If the system is prepared in its ground state at the beginning of the quenching, i.e., $C_{1}(\mathbf{k})=1$ at $t=-\infty$, the above equation suggests that the probability of the nonadiabatic transition, $p_{\mathbf{k}}=\lim _{t \rightarrow+\infty}\left|C_{1, \mathbf{k}}\right|^{2}$, must have a functional dependence on $|\Delta(\mathbf{k})|^{2} \tau$ of the form

$$
p_{\mathbf{k}}=f\left(|\Delta(\mathbf{k})|^{2} \tau\right) .
$$

The analytical form of the function $f$ is given by the general LZ formula [37, 40]. The defect density in the final state is therefore given by [31]

$$
n=\int \frac{d^{d} k}{(2 \pi)^{d}} f\left(|\Delta(\mathbf{k})|^{2} \tau\right)=\int \frac{d^{d} k}{(2 \pi)^{d}} f\left(|\mathbf{k}|^{2 z_{2}} \tau\right) .
$$

The scaling $|\mathbf{k}| \rightarrow|\mathbf{k}|^{2 z_{2}} \tau$ finally leads to a scaling of the defect density given by

$$
n \sim 1 / \tau^{d /\left(2 z_{2}\right)} .
$$

Let us recall the quenching dynamics of the transverse $X Y$ spin chain discussed before [13, 16]. When the system is quenched across the Ising or anisotropic critical line by linearly changing $h$ or $J_{x}$ as $t / \tau, \Delta(\mathbf{k})$ vanishes at the critical point as $\Delta(\mathbf{k}) \sim|\mathbf{k}|$ yielding $z_{2}=z=1$; hence the generalized scaling form given in Eq. (1.28) matches with the Kibble-Zurek prediction with $v=z=1$ as mentioned before. The situation, however, is different when the system is swept across a multicritical point.

Putting $h=2 J_{y}$ in the Hamiltonian in (1.15), let us analyze the scaling of the diagonal and off-diagonal terms of the Hamiltonian at the multicritical point. When $J_{x}=J_{y}$, the diagonal term of the Hamiltonian scales as $-J_{y}(\pi-k)^{2}$ whereas the offdiagonal term $|\Delta(k)| \sim|\pi-k|^{3}$ when expanded around the critical mode at $k=\pi$. The dynamical exponent is obtained from the diagonal term so that $z=z_{1}=2$. As discussed above, the off-diagonal term or more precisely the exponent $z_{2}$ determines the scaling of the defect density. The exponent $z_{2}=3$ for the $X Y$ multicritical point; hence the defect density scales as $1 / \tau^{1 / 6}$. The density of defects obtained by numerical integration of the Schrödinger equation in (1.23) as shown in Fig. 1.6 supports this scaling behavior. For a non-linear quench across a multicritical point [36], when the parameter $\lambda$ is quenched as $\lambda \sim(t / \tau)^{\alpha} \operatorname{sgn}(t)$, both $z_{1}$ and $z_{2}$ come into play and the scaling of defect density gets altered to $n \sim \tau^{-d \alpha v /\left[\alpha\left(z_{2} v+1\right)+z_{1} v(1-\alpha)\right]}$; this reduces to the form presented above for $\alpha=1$ and $z_{2} v=1$. 


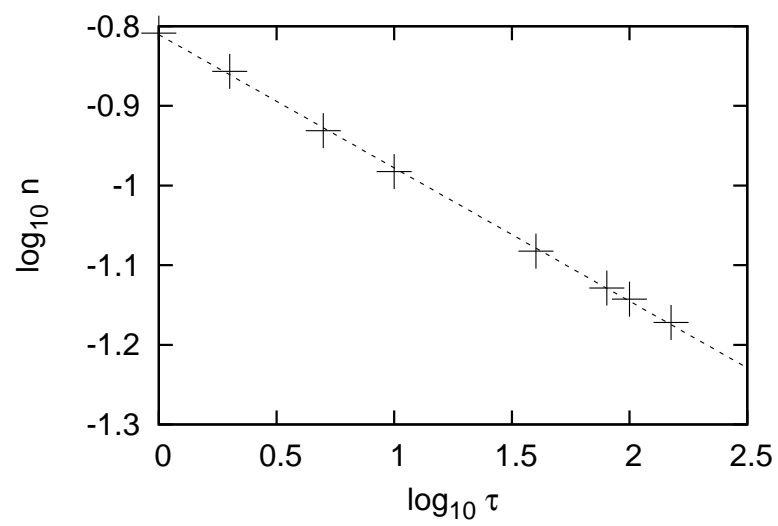

Fig. 1.6. $n$ vs $\tau$ obtained by numerically solving Eq. 1.23 at the multicritical point of the $X Y$ model in a transverse field, with $h=10$ and $J_{y}=5$. The line has a slope of -0.16 .

\subsection{Quenching along a gapless line}

We now shift our focus to quenching the $X Y$ Hamiltonian in (1.7) along the gapless line $h=J_{x}+J_{y}$ by varying the anisotropy parameter $\gamma=J_{x}-J_{y}$ as $t / \tau$, keeping $J_{x}+J_{y}$ fixed [26]. For convenience, let us set $J_{x}+J_{y}=h=1$. The excitation gap vanishes along this line for the mode $k=\pi$. We rewrite Eq. (1.8) with the present notation in the form

$$
H_{k}=\left[\begin{array}{ll}
1+\cos k & i \gamma \sin k \\
-i \gamma \sin k & -1-\cos k
\end{array}\right] .
$$

We once again encounter a situation in which the off-diagonal terms are timedependent. Noting that the asymptotic form of the Hamiltonian at $t \rightarrow \pm \infty$ is given by

$$
H=\frac{t}{\tau} \sin k \hat{\sigma}^{x},
$$

we make a basis transformation to a representation in which $\sigma^{x}$ is diagonal. The Hamiltonian in (1.29) then gets modified to the form

$$
\left[\begin{array}{ll}
(t / \tau) \sin k & 1+\cos k \\
1+\cos k & -(t / \tau) \sin k
\end{array}\right]
$$

where the time dependence has been shifted to the diagonal terms only. Applying the LZ transition probability formula, the probability of excitations is found to be

$$
p_{k}=e^{-\pi \tau\left|\Delta_{k}\right|^{2} / \sin k}
$$

where $\left|\Delta_{k}\right|^{2}=|1+\cos k|^{2}=(\pi-k)^{4} / 4$ when expanded about $k=\pi$. Integrating $p_{k}$ over the Brillouin zone, we find the that density of defects falls off as $n \sim 1 / \tau^{1 / 3}$. 
Fig. 1.7 which shows $n$ vs $\tau$ obtained by numerical integration of the Schrödinger equation confirms the $\tau^{-1 / 3}$ behavior.

It is to be noted that the Kibble-Zurek formalism cannot address defect generation along a gapless line. We will now present another general scaling argument for moving along a gapless line in a $d$-dimensional system.

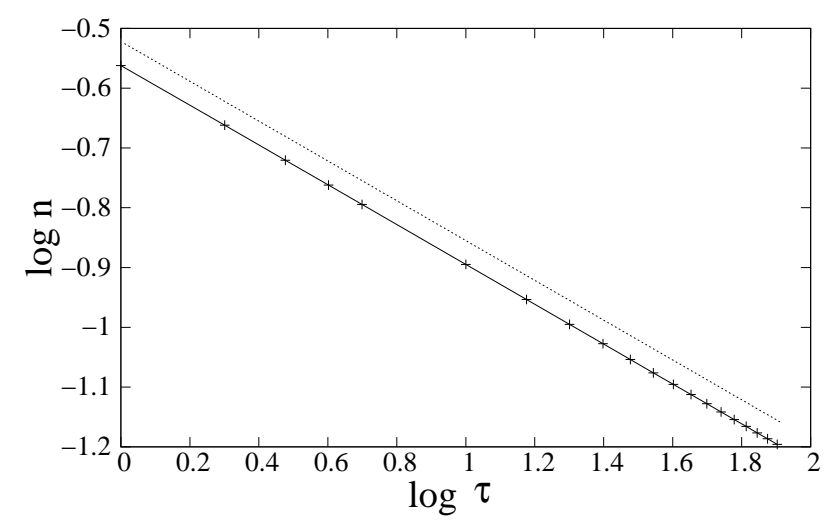

Fig. 1.7. $n$ vs $\tau$ obtained by numerically solving Schrödinger equation with $\gamma(t) \sim t / \tau$ keeping $h=2 J_{y}$. Also shown is a line with slope $1 / 3$ for comparison.

Let the excitations on the gapless quantum critical line be of the form $\omega_{\mathbf{k}} \sim \lambda|\mathbf{k}|^{z}$, where $z$ is the dynamical exponent and the parameter $\lambda=t / \tau$ is quenched from $-\infty$ to $\infty$. Using a perturbative method involving the Fermi Golden rule along with the fact that the system is initially prepared in the ground state, the defect density can be approximated as [19]

$$
n \simeq \int \frac{d^{d} k}{(2 \pi)^{d}}\left|\int_{-\infty}^{\infty} d \lambda\left\langle\mathbf{k}\left|\frac{\partial}{\partial \lambda}\right| 0\right\rangle e^{i \tau \int^{\lambda} \delta \omega_{\mathbf{k}}\left(\lambda^{\prime}\right) d \lambda^{\prime}}\right|^{2} .
$$

Let us assume a general scaling form for the instantaneous excitation, $\delta \omega_{\mathbf{k}}\left(\lambda^{\prime}\right)=$ $k^{a} f\left(\frac{\lambda k^{z}}{k^{a}}\right)$, where $k=|\mathbf{k}|$ and $k^{a}$ denotes the higher order term in the excitation spectrum on the gapless line. Defining a new variable $\xi=\lambda k^{z-a}$, we obtain the scaling behavior of the defect density as [26]

$$
n \sim 1 / \tau^{d /(2 a-z)} .
$$

The case $d=1, a=2$ and $z=1$ has been discussed in this section. Note that the correlation length exponent $v$ does not appear in the expression in Eq. (1.34) because our quench dynamics always keeps the system on a critical line. The scaling for quenching along a gapless line is less universal in comparison to the general KibbleZurek prediction. For a non-linear quench [22] $\lambda \sim(t / \tau)^{\alpha} \operatorname{sgn}(t)$, this scaling form gets modified to $n \sim \tau^{-d \alpha /[a(\alpha+1)-z]}$; this reduces to $1 / \tau^{1 / 3}$ for $\alpha=d=z=1$ and $a=2$. 
In passing, let us introduce a variant of the $X Y$ Hamiltonian with an alternating field given by [42, 28]

$$
\begin{aligned}
H=-\frac{1}{2}[ & \sum_{j}\left(J_{x}+J_{y}\right)\left(\sigma_{j}^{x} \sigma_{j+1}^{x}+\sigma_{j}^{y} \sigma_{j+1}^{y}\right) \\
& \left.+\left(J_{x}-J_{y}\right)\left(\sigma_{j}^{x} \sigma_{j+1}^{x}-\sigma_{j}^{y} \sigma_{j+1}^{y}\right)+\left(h-(-1)^{j} \delta\right) \sigma_{j}^{z}\right] .
\end{aligned}
$$

The strength of the transverse field alternates between $h+\delta$ and $h-\delta$ on the odd and even sites respectively. For $\delta=0$, we recover the conventional $X Y$ model in a transverse field. This Hamiltonian can also be solved exactly by a Jordan-Wigner transformation by taking care of the two underlying sublattices, i.e., by defining $a_{k}$ and $b_{k}$ as two different Jordan-Wigner fermions. The Hamiltonian in the basis $\left(a_{k}^{\dagger}, a_{-k}, b_{k}^{\dagger}, b_{-k}\right)$ can be written as

$$
H_{k}=\left[\begin{array}{cccc}
h+J \cos k & i \gamma \sin k & 0 & -\delta \\
-i \gamma \sin k & -h-J \cos k & \delta & 0 \\
0 & \delta & J \cos k-h & i \gamma \sin k \\
-\delta & 0 & -i \gamma \sin k & -J \cos k+h
\end{array}\right]
$$

The excitation spectrum of the above Hamiltonian is

$$
\begin{aligned}
\Lambda_{k}^{ \pm}= & {\left[h^{2}+\delta^{2}+J^{2} \cos ^{2} k+\gamma^{2} \sin ^{2} k\right.} \\
& \left. \pm 2 \sqrt{h^{2} \delta^{2}+h^{2} J^{2} \cos ^{2} k+\delta^{2} \gamma^{2} \sin ^{2} k}\right]^{1 / 2},
\end{aligned}
$$

where $J=J_{x}+J_{y}$ and $\gamma=J_{x}-J_{y}$, with four eigenvalues given by $\pm \Lambda_{k}^{ \pm}$. In the ground state, $-\Lambda_{k}^{+}$and $-\Lambda_{k}^{-}$are filled. The vanishing of $\Lambda_{k}^{-}$dictates the quantum critical point, and the critical exponents are obtained by studying the behavior of $\Lambda_{k}^{-}$in the vicinity of the critical point. The minimum energy gap in the excitation spectrum occurs at $k=0$ and $k=\pi / 2$. The corresponding phase boundaries $h^{2}=\delta^{2}+J^{2}$ and $\delta^{2}=h^{2}+\gamma^{2}$ signal quantum phase transitions from a paramagnetic to a ferromagnetic phase respectively. We extend the study of quenching through a gapless phase in this Hamiltonian by varying $\gamma$ as before along the phase boundary $h^{2}=\delta^{2}+J^{2}$. On this gapless line, the dispersion of the low-energy excitations at $k \rightarrow 0$ can be approximated as

$$
\Lambda_{k}^{-}=\sqrt{\frac{J^{4} k^{4}}{4\left(\delta^{2}+J^{2}\right)}+\frac{\gamma^{2} J^{2} k^{2}}{\delta^{2}+J^{2}}} .
$$

This suggests a truncation of the Hamiltonian in $(1.36$ to a $2 \times 2$ LZ Hamiltonian

$$
h_{k}=\left[\begin{array}{cc}
\tilde{\gamma}(t) k & \tilde{J}^{2} k^{2} / 2 \\
\tilde{J}^{2} k^{2} / 2 & -\tilde{\gamma}(t) k
\end{array}\right]
$$

where $\tilde{\gamma}$ and $\tilde{J}$ are renormalized parameters given by $\tilde{\gamma}=\gamma J / \sqrt{\delta^{2}+J^{2}}$ and $\tilde{J}^{2}=$ $J^{2} / \sqrt{\delta^{2}+J^{2}}$. The argument which justifies the truncation of a $4 \times 4$ matrix to a $2 \times 2$ matrix is presented in Ref. [26]. The diagonal terms in Eq. (1.39) describe 
two time-dependent levels approaching each other linearly in time (since $\gamma=t / \tau$ ), while the minimum gap is given by the off-diagonal term $\tilde{J}^{2} k^{2} / 2$. The probability of excitations $p_{k}$ from the ground state to the excited state for the $k$-th mode is given by the LZ transition formula [40, 37]

$$
p_{k}=\exp \left[-\frac{2 \pi \tilde{J}^{4} k^{4}}{8 k d \tilde{\gamma}(t) / d t}\right]=\exp \left[-\frac{\pi J^{3} k^{3} \tau}{4 \sqrt{\delta^{2}+J^{2}}}\right]
$$

and we get back the $\tau^{-1 / 3}$ scaling form of the defect density as discussed above.

\subsection{Conclusions}

The non-equilibrium dynamics of a quantum system driven through a quantum critical point is indeed an exciting and fascinating area of research. The possibility of experimental realizations of quenching dynamics adds to the importance of the theoretical research. In this review we have discussed the scaling relations for the defect density in the final state following a slow quench across a multicritical point and along a gapless line. In both cases, the relations obtained here using the LZ transition formula are not directly derivable using the Kibble-Zurek argument. But in all the cases, the defect density scales as a power law with the rate of quenching. It should be noted that the idea of a dominant critical point has also been invoked to justify the scaling along a gapless line in one-dimensional spin models [28]. A generalization of the studies presented here to higher dimensional quantum systems and to systems with quenched disorder are some of the future directions of this subject.

\section{Acknowledgements}

The authors acknowledge the organizers of the conference "Quantum Annealing and Quantum Computation". We also thank A. Polkovnikov, S. Sachdev, G. E. Santoro and K. Sengupta for useful discussions at different occasions. AD also acknowledges R. Moessner and hospitality of MPIPKS, Dresden, where some part of the work discussed in the review was done.

\section{References}

1. S. Sachdev, Quantum Phase Transitions (Cambridge University Press, Cambridge, 1999).

2. B. K. Chakrabarti, A. Dutta and P. Sen, Quantum Ising Phases and Transitions in Transverse Ising Models, m41 (Springer-Verlag, Berlin, 1996).

3. K. Sengupta, S. Powell and S. Sachdev, Phys. Rev. A 69, 053616 (2004); A. Das, K. Sengupta, D. Sen and B. K. Chakrabarti, Phys. Rev. B 74, 144423 (2006).

4. W. H. Zurek, U. Dorner and P. Zoller, Phys. Rev. Lett. 95, 105701 (2005); B. Damski, Phys. Rev. Lett. 95, 035701 (2005).

5. A. Polkovnikov, Phys. Rev. B 72, 161201(R) (2005). 
6. L. E. Sadler, J. M. Higbie, S. R. Leslie, M. Vengalattore and D. M. Stamper-Kurn, Nature 443, 312 (2006).

7. L.-M. Duan, E. Demler and M. D. Lukin, Phys. Rev. Lett. 91, 090402 (2003); A. Micheli, G. K. Brennen and P. Zoller, Nature Physics 2, 341 (2006); E. Altman, E. Demler and M. D. Lukin, Phys. Rev. A 70, 013603 (2004).

8. I. Bloch, J. Dalibard and W. Zwerger, Rev. Mod. Phys. 80, 885 (2008).

9. T. Kadowaki and H. Nishimori, Phys. Rev. E 58, 5355 (1998).

10. P. Calabrese and J. Cardy, J. Stat. Mech: Theory Expt P04010 (2005), and Phys. Rev. Lett. 96, 136801 (2006); R. Schützhold, M. Uhlmann, Y. Xu and U. R. Fischer, Phys. Rev. Lett. 97, 200601 (2006); C. Kollath, A. M. Läuchli and E. Altman, Phys. Rev. Lett. 98, 180601 (2007); S. R. Manmana, S. Wessel, R. M. Noack and A. Muramatsu, Phys. Rev. Lett. 98, 210405 (2007); M. Eckstein and M. Kollar, Phys. Rev. Lett. 100, 120404 (2008).

11. J. Dziarmaga, Phys. Rev. Lett. 95, 245701 (2005).

12. B. Damski and W. H. Zurek, Phys. Rev. A 73, 063405 (2006).

13. R. W. Cherng and L. S. Levitov, Phys. Rev. A 73, 043614 (2006).

14. J. Dziarmaga, Phys. Rev. B 74, 064416 (2006); T. Caneva, R. Fazio and G. E. Santoro, Phys. Rev. B 76, 144427 (2007).

15. F. M. Cucchietti, B. Damski, J. Dziarmaga and W. H. Zurek, Phys. Rev. A 75, 023603 (2007).

16. V. Mukherjee, U. Divakaran, A. Dutta and D. Sen, Phys. Rev. B 76, 174303 (2007).

17. U. Divakaran and A. Dutta, J. Stat. Mech: Theory and Experiment P11001 (2007).

18. A. Fubini, G. Falci and A. Osterloh, New J. Phys 9, 134 (2007).

19. A. Polkovnikov and V. Gritsev, Nature Physics 4, 477 (2008).

20. K. Sengupta, D. Sen and S. Mondal, Phys. Rev. Lett. 100, 077204 (2008); S. Mondal, D. Sen and K. Sengupta, Phys. Rev. B 78, 045101 (2008).

21. D. Sen, K. Sengupta and S. Mondal, Phys. Rev. Lett. 101, 016806 (2008); R. Barankov and A. Polkovnikov, Phys. Rev. Lett. 101, 076801 (2008); C. De Grandi, R. A. Barankov and A. Polkovnikov, Phys. Rev. Lett. 101, 230402 (2008).

22. S. Mondal, K. Sengupta and D. Sen, Phys. Rev. B 79, 045128 (2009).

23. F. Pellegrini, S. Montangero, G. E. Santoro and R. Fazio, Phys. Rev. B 77, 140404(R) (2008).

24. D. Patanè, A. Silva, L. Amico, R. Fazio and G. E. Santoro, Phys. Rev. Lett. 101, 175701 (2008).

25. V. Mukherjee, A. Dutta and D. Sen, Phys. Rev. B 77, 214427 (2008).

26. U. Divakaran, A. Dutta and D. Sen, Phys. Rev. B 78, 144301 (2008).

27. T. Caneva, R. Fazio and G. E. Santoro, Phys. Rev. B 78, 104426 (2008).

28. S. Deng, G. Ortiz and L. Viola, Europhys. Lett. 84, 67008 (2008).

29. J. Dziarmaga, J. Meisner and W. H. Zurek, Phys. Rev. Lett. 101, 115701 (2008).

30. A. Bermudez, D. Patanè, L. Amico and M. A. Martin-Delgado, Phys. Rev. Lett. 102, 135702 (2009).

31. U. Divakaran, V. Mukherjee, A. Dutta and D. Sen, J. Stat. Mech: Theory and Expt. P02007 (2009).

32. V. Mukherjee and A. Dutta, J. Stat. Mech: Theory and Experiment P05005 (2009).

33. U. Divakaran and A. Dutta, arXiv:0901.3260 to appear in Phys. Rev. B (2009).

34. T. W. B. Kibble, J. Phys. A 9, 1387 (1976), and Phys. Rep. 67, 183 (1980).

35. W. H. Zurek, Nature 317, 505 (1985), and Phys. Rep. 276, 177 (1996).

36. S. Mondal, D. Sen and K. Sengupta, Non-equilibrium dynamics of quantum systems: order parameter evolution, defect generation, and qubit transfer, in these Lecture Notes.

37. S. Suzuki and M. Okada, in Quantum Annealing and Related Optimization Methods, Ed. by A. Das and B. K. Chakrabarti (Springer-Verlag, Berlin, 2005), p. 185. 
38. J. E. Bunder and R. H. McKenzie, Phys. Rev. B 60, 344 (1999).

39. E. Lieb, T. Schultz and D. Mattis, Ann. Phys. 16, 407 (1961); E. Barouch and B. M. McCoy, Phys. Rev. A 3, 786 (1971); J. B. Kogut, Rev. Mod. Phys. 51, 659 (1979).

40. C. Zener, Proc. Roy. Soc. London Ser A 137, 696 (1932); L. D. Landau and E. M. Lifshitz, Quantum Mechanics: Non-relativistic Theory, 2nd ed. (Pergamon Press, Oxford, 1965).

41. A. Kitaev, Ann. Phys. 321, 2 (2006).

42. J. H. H. Perk, H. W. Capel and M. J. Zuilhof, Physica 81 A, 319 (1975); K. Okamoto and K. Yasumura, J. Phys. Soc. Jap. 59, 993 (1990). 